\title{
Implementation of SBRT as a Rescue Technique for Reirradiation of Local Recurrence of Prostatic Cancer
}

\author{
Mariana Perez De Antueno* \\ Medical Specialist in Radiotherapy, Radiotherapy service, Argentina
}

*Corresponding author: Mariana Perez De Antueno, Medical Specialist in Radiotherapy, Radiotherapy service, Argentina.

Received Date: June 08, 2020

Published Date: July 01, 2020

\begin{abstract}
Background: External beam radiation therapy and brachytherapy are very efficient treatment modalities for localized prostate cancer, although more than $30 \%$ of the patients would experience a biochemical recurrence within 10 years. Among these patients, about $20 \%$ would present a local recurrence, and optimal management of locally recurrent prostate cancer after definitive radiation therapy is still challenging. In this scenario the most common retreatment option is androgen deprivation therapy, which cannot be considered as a radical treatment. Surgery, as a radical salvage modality and other focal treatments such as cryotherapy, high-intensity focused ultrasound (HIFU), and brachytherapy can be offered to highly selected patients. More recently, the development of SBRT (stereotactic body radiation therapy), allows to deliver higher ablative dose of radiation to a smaller target volume with better sparing of surrounding critical organs at risk and can be an encouraging alternative for patients with a postirradiation relapse of prostate cancer. To date, the number of publications in this fields considerably low, and results are inconclusive mainly due to the limited number of patients treated and the lack of randomized clinical trials.
\end{abstract}

Hypothesis: The intensification of focal doses of radiation with SBRT, in a rescue scenario after a post-irradiation relapse of prostate cancer, can be a safe and effective local treatment.

Objectives: Primary: The purpose of this research study is to implement SBRT for the treatment of post- irradiated local recurrence prostate cancer. Secondary: The aim of this study was to evaluate the tolerance and the effectiveness (biochemical control, disease free survival and survival) of SBRT in previously irradiated prostate cancer relapses.

Material, Methods and Stages of development: A) Selection of the patients at the multidisciplinary tumor board. Eligibility criteria: Patients with a biochemical recurrence (PSA level $>2 \mathrm{ng} / \mathrm{ml}$ ) and a proven local recurrence of prostate cancer previously irradiated (proven by biopsy or positive 18 F-Choline- PET- CT or PSMA PET-TC). With good performance status and absence of previous rectal or urinary radiation induced toxicity (normal cystoscopy and rectoscopy).

B) Establish treatment protocol: CT simulation and immobilization will be described. GTV, CTV and PTV margins, total dose, fractionation and constraints of organs at risk will be defined. IMRT or VMAT will be performed with daily IGRT.

C) Follow up to evaluate toxicity, local control and survival will be performed every 3 months during the first 2 years. Evaluation of clinical tolerance and clinical response will be performed, with a quality of life questionnaire, and PSA levels. 18 F-Choline PET-CT or PSMA PET-TC will be performed in case of biochemical failure (PSA levels $>2 \mathrm{ng} / \mathrm{ml}$ )

D) Previous approval of the Local Research Ethics Committee.

\section{Background}

Prostate cancer is the most common malignancy diagnosed in men. Treatment options for prostate cancer are based on staging the risk score with Gleason, clinical stage, and PSA. These treatment options are varied, one of which is radiation therapy [1]. These therapies offer excellent long-term cure rates in men with localized prostate cancer, but $30 \%$ to $50 \%$ of men will have evidence of 
biochemical recurrence of PSA disease 10 years after treatment by any form of therapy [2]. Recurrence of the disease occurs most frequently in the form of biochemical recurrence, with an isolated, detectable increasing PSA level and no other clinical evidence of the disease [2,3] (Figure 1).

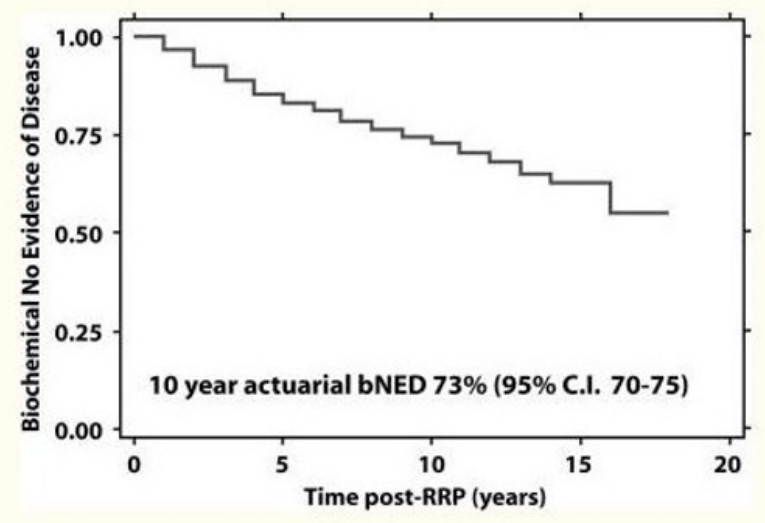

Figure 1: Graph.

In those patients who were previously irradiated as primary therapy for localized prostate cancer and who unfortunately have local prostatic relapse, there are different alternatives for retreatment. The most common is probably Androgen Deprivation Therapy (ADT), which cannot be considered as radical treatment. When we consider radical rescue modalities, surgery seems to be the treatment of choice and for a considerable period of time it was the only option. Later, new possibilities such as cryotherapy, high intensity focused ultrasound (HIFU), and brachytherapy (BT) appeared [4]. All the aforementioned modalities have yielded promising but inconsistent results, mainly due to limited availability, the state of performance of the patient and/or comorbidities, local anatomical conditions, and lack of patient agreement [4]. More recently, the development of SBRT (Stereotactic Body Radiotherapy) which allows a higher ablative dose of radiation to be delivered at a smaller target volume, with better preservation of the surrounding critical organs at risk, could be an encouraging alternative, for patients with a local prostate relapse who has already received prior radiant treatment in that organ [5]. Of interest to the radiation oncologist, prostate cancer has an $\alpha$ / $\beta$ ratio $<2$ that offers potential therapeutic benefit for hypofractionation such as SBRT, which may be more advantageous compared to conventional regimens because this low $\alpha / \beta$ ratio prostate cancer thus offers high dose sensitivity per fraction. Furthermore, the higher estimated $\alpha / \beta$ ratio of the rectum and a smaller, tighter margin with SBRT provide acceptable toxicity despite the higher dose per fraction [1-6].

\section{PLANIFICACION SBRT}

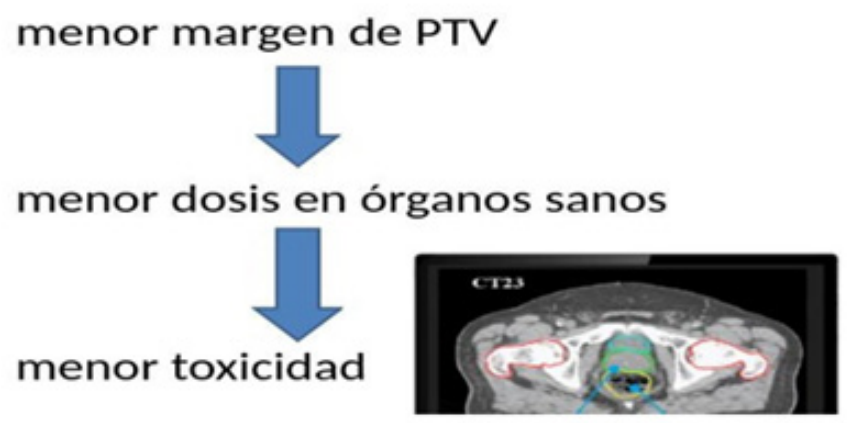

Figure 2: SBRT planning.

Although rethinking irradiation for local recurrence in patients who have already received primary radiation therapy represents a challenging option, since a basic and long-lasting principle of radiation therapy is that once definitive RT has been administered, additional RT cannot be administered. because it would probably exceed normal tissue tolerances. The enormous technological advances from diagnosis to treatment planning and administration of radiotherapy have sparked interest in evaluating the suitability 
of re-irradiation in the treatment of recurrent PCa. In this context, brachytherapy allows a flexible radiation dose, it is expected to deliver a relatively small dose to the surrounding tissues, which has the potential for better rectal savings compared to conventional external radiation therapy (EBRT). This has recently prompted the Radiation Therapy Oncology Group (RTOG) to evaluate the role of brachytherapy for locally recurrent prostate adenocarcinoma in an ongoing prospective phase II trial (RTOG 0526). However, the main drawback is that brachytherapy is an invasive procedure associated with not insignificant risks of infection and thromboembolism, which makes its choice not suitable for many patients. Meanwhile, early data consistently shows that stereotactic body radiation therapy (SBRT) is a safe and effective treatment modality in the reirradiation situation for various anatomical sites [7]. SBRT allows for steep dose gradients that resemble those brachytherapy dose distributions, with the added benefits of avoiding hospitalization, catheterization, and discomfort from inserted needles over a long period of time (in the case of LDR implants) or the need to monitor permanent transperineal HDR catheters and dealing with its accompanying pain. Therefore, highly focused radiation beams can minimize radiation-associated rectal toxicity by reducing the volume of the rectum receiving high doses of radiation [7]. Furthermore, taking advantage of the favorable therapeutic ratio offered by the low $\alpha$ / $\beta$ ratio of prostate cancer, SBRT enables a dramatic reduction in the number of fractions and overall treatment time, making irradiation possible with few dose fractions. very big. Delivering such a treatment regimen certainly requires the adoption of techniques with multiple non-coplanar fields or intensity modulated arcotherapy [7] (Figure 2).

Regarding toxicity, the study by Stefano Arcangeli, which attempts to investigate predictors of acute gastrointestinal (GI) and genitourinary (GU) toxicity after a short-cycle hypofractionated radiotherapy regimen for prostate cancer, three institutions included 102 patients with T1-T3N0M0 prostate cancer in a phase II study. The patients were treated with 56 Gy in 16 fractions for 4 weeks. Acute toxicity was rated weekly during treatment and 1 and 2 months after treatment using the criteria of the Extended Radiation Oncology Group / European Organization for Research and Treatment of Cancer with additional symptoms and the International Prostate Symptom Index (IPSS). They observed no grade 3 or 4 gastrointestinal side effects. Grades 1 and 2 of rectal GI toxicity occurred in 36\% and 38\%, respectively. The corresponding figures for GU toxicity grades 1 and 2 were $42 \%$ and $39 \%$, respectively. GU toxicity grade 3 or higher was detected in $4 \%$ of patients. In multivariate analysis, percentage rectal volumes greater than $8 \%$ receiving doses $\geq 53$ Gy (V 53) were statistically correlated with Grade 2 acute rectal reaction $(p=0.006)$. For GU morbidity, only the IPSS pretreatment score was independently associated ( $p=0.0036)$ with increased acute effects of GU. In conclusion they mention that the acute toxicity GU and GI were comparable with other series. Their data shows that the increased incidence and intensity of acute toxicity is a transient effect related to a shorter overall treatment time rather than a greater effect on the biological equivalent dose over a conventional fractionation regimen. In patients previously irradiated and with local prostate relapse, where SBRT was used as a reirradiation technique, it is reported that urinary toxicity included five acute events (only one grade 3) and three late events (only one grade 3) and consider SBRT As a feasible approach for isolated intraprostatic ecurrence, which offers excellent control in previously irradiated patients with local prostate relapse, where SBRT was used as a reirradiation technique, it states that urinary toxicity included five acute events (only one grade 3) and three late events (only one grade 3) and consider SBRT as a feasible approach to isolated intraprostatic recurrence, offering excellent local tumor control and a low toxicity profile [7-13].

In addition, re-irradiation of the prostate with the SBRT technique allows reducing the dose to the ectum, which has already received high doses with primary treatment. In the study carried out by Jereczek Fossa, from the Italian group of the European Institute of Oncology IRCCS, the acute and chronic toxicity of gastrointestinal (GI) and genitourinary (GU) events of 64 patients, who received a mean total dose of $30 \mathrm{~Gy}$, were evaluated in 5 fractions, with a BED of 150Gy, objectifying 1 acute G3 genitourinary (GU) event and 1 late GU G3 event [14]. There was no G $\geq 3$ intestinal toxicity. At the mean follow-up of 26.1 months, progression was observed in 41 patients (64\%) and 18 patients (28\%) experienced a local relapse. Two-year local control, biochemical and clinical relapse-free survival rates were $75 \%, 40 \%$, and $53 \%$, respectively. With BED $\geq 130$ Gy, the one-year clinical and biochemical progression-free survival rate was $85 \%$ and $90 \%$, respectively [14]. Retrospective cohort studies report favorable morbidity to SBRT re-irradiation of the prostate, but the studies are small and the follow-up is short. These studies indicate that the risk lies in GU side effects, rather than gastrointestinal effects, and that careful selection of patients without pre-existing morbidity GU and tight dose restrictions to the urethra and bladder neck are important for limit the risk of GU morbidity. Therefore, more studies and prolonged follow-ups are necessary to conclude on the effect of SBRT re-irradiation in the control of local recurrence of prostate cancer after irradiation.

\section{Proposal of the Study}

1. In this observational and prospective study, SBRT (body stereotactic radiotherapy) will be developed as a rescue technique for reirradiation, in patients with local relapse of prostate cancer (PCa), in the Radiotherapy Service of the Hospital in Argentina, with the goal of being able to achieve this goal, safely and precisely, with state-of-the-art linear accelerators, but without a cyberknife. 
2. The secondary objective will be to review tolerance and effectiveness (biochemical control, disease-free survival and survival) in patients with previously irradiated prostate cancer relapse who were treated with SBRT as a re-irradiation technique.

\section{Selection of Patients}

The selection of the patients is made in the Committee of Experts in SBRT and Radiosurgery of the Hospital where each case is presented and reviewed in a particular way.

\section{Eligibility criteria}

1. Patients with a biochemical relapse, that is, PSA nadir +2 $\mathrm{ng} / \mathrm{ml}$, according to what is established by the Radiation Therapy Oncology Group (RTOG) and American Society for Radiation Oncology (ASTRO) Phoenix Consensus [8] and evidence of isolated local recurrence proven, of prostate cancer, by biopsy or positivity of multiparametric prostate NMR (mp NMR) or PET-TC 18 F-choline or PET -TC PSMA. (Biopsy is not mandatory if all the diagnostic elements were unambiguous, such as the evolution of prostate-specific antigen and imaging study such as mpRMN or PET-CT).

2. Patient previously irradiated with external radiotherapy or brachytherapy

3. Good functional state and absence of toxicity induced by previous rectal or urinary radiation (normal cystoscopy and rectoscopy)

4. Completion of the IPSS prior to the start of the practice

5. Signature of written informed consent for re-irradiation with SBRT technique.

\section{Exclusion criteria}

1. Patients with biopsy and mpRMN, choline PET-TC or PSMA-negative PET, which does not allow locating the focus of relapse.

2. Pathologies in the bladder, urethra or rectum, either as a base or as a sequel to previously received radiotherapy, such as urethral stricture due to previous transurethral resection (TURP), proctitis, radioinduced rectitis, diverticulitis, among others.

\section{Radiotherapy Treatment}

\section{Preparation and conditioning of the patient}

All patients will be asked orally and in writing for the following rectal and bladder preparation.

1. Instructions for low residue diet

2. The taking of simethicone suspension from 48 hours prior to the simulation CT will be indicated.
3. A sodium phosphate enema $(250 \mathrm{ml})$ will be placed to clean the rectum before the simulation and before each treatment fraction.

4. It will be requested to have the complete urinary bladder, for this it is indicated that the patients drink 600-700 cc of water (approximately 3 full glasses of water) half an hour before the tomography.

5. Every day of treatment, the same preparation is requested.

\section{Simulation Tomography (CAT)}

1. It will be carried out with the above preparation the patient will be placed in a supine position on an immobilizer for the pelvis and lower extremities to perform the SBRT technique. Thermoplastic immobilization and respiratory control system are not required.

2. The patient will be aligned with the 3 corresponding lasers, one located on the ceiling and two on the patient's left and right sides.

3. The requested tomographic cuts are made every $3 \mathrm{~mm}$.

4. These characteristics performed in the Simulation CT are specified and explained in the patient's medical history in order to have these data in each of the treatment sessions.

\section{Volume contouring}

1. The outline of the gross tumor volume (GTV) will be based on mpMRI or PET-CT, images that must be merged, thanks to the Eclipse ${ }^{\text {тм }}$ Treatment Planning System ((Varian Medical Systems, Palo Alto, California, distributed by Photonix, Autonomous City of Buenos Aires - Argentina) with simulation tomography.

2. The clinical target volume (CTV) will include the area of recurrence of intraprostatic lesion with margins.

3. The margins of CTV to plan the target volume (PTV) will be $5 \mathrm{~mm}$ in all directions except the posterior margin where the margin is corrected to $3 \mathrm{~mm}$. For treatments in which the interval between both RT courses is short, comorbidities or unfavorable patient anatomy, or unfavorable dose volume histograms (DVH) data, margins can be reduced to $1 \mathrm{~mm}$ or 2 $\mathrm{mm}$ in all directions. . This decision is supported by the strong dose gradient that can be achieved with the VMAT dosimetry and with the verification using the CT cone-beam image of the Trilogy ${ }^{\circledR}$ accelerator, which is performed prior to each of the treatment applications. It helps determine the positioning of the internal organs.

4. Planning of the volume of organs at risk (PRV). A urethral PRV is performed: $3 \mathrm{~mm}$ extension around the urethra (i.e. urethra + PRV) 
5. Organs at risk (OAR) included the rectum (and its posterior part), urinary bladder, urethra, penis bulb, penis, testis, femoral heads, cavity peritoneal and cauda equina which, thanks to the fusion performed with the mpMRI, are delimited with greater accuracy. OARs should be contoured following the consensus of RTOG contouring of the normal tissue of the male pelvis [12].

\section{Treatment Scheme}

Extreme hypofractionation will be used. The dose protocol to be used is the most frequently used in the literature: 30 Gy in 5 fractions of 6 Gy to PTV. The dose is prescribed at $95 \%$ and is administered every other day [11]. We have chosen the fractionation of 30 Gy into 5 fractions of 6 Gy, because taking into account the $\alpha / \beta<2$ of the Table 1: Dose and volume restrictions prostate, the BED (Biologically Effective Dose of radiation) is $>130$ Gy [12] and in published studies they found Statistically significant differences for the one-year progression-free biochemical survival rate $(85 \%$ vs. $60 \%$, p-value $=0.0006)$ and the one-year clinical progression-free survival rate $(90 \%$ vs. $73 \%$, p-value $=0.0026)$ and rescue SBRT reirradiation for locally recurrent PCa offers satisfactory tumor control and an excellent toxicity profile, if BED $\geq 130$ Gy is administered and patients are appropriately selected [14].

\section{Normal Tissue-Volume Restrictions}

The dose and volume restrictions that we will use are the following [13-14] (Table 1)

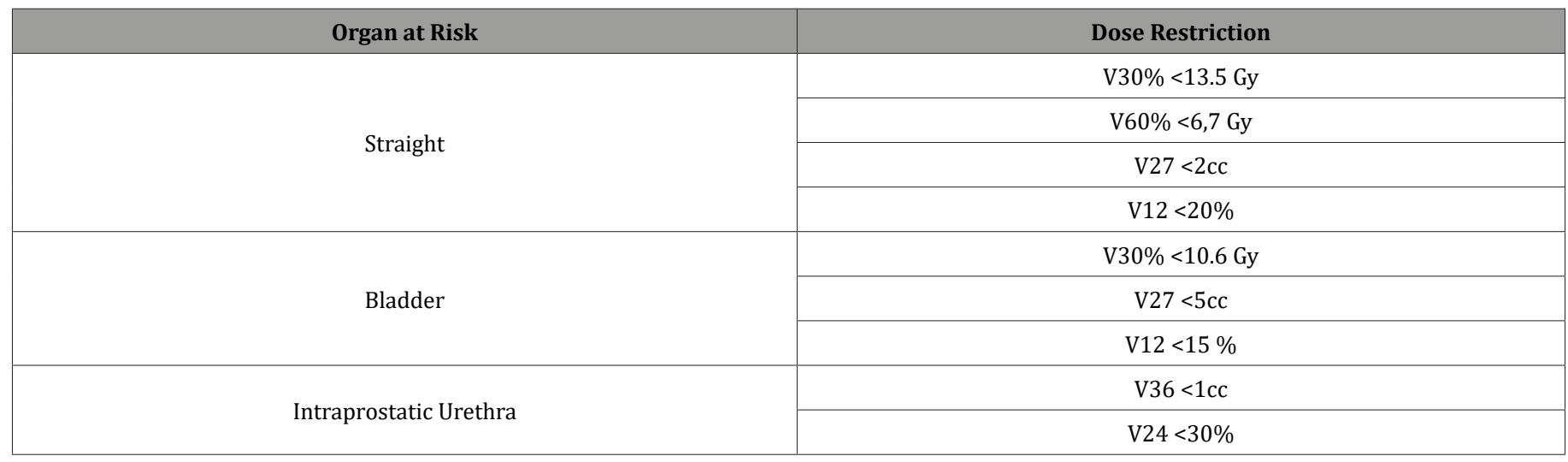

In this table you can see the dose restrictions of the different of the organ to be evaluated can receive will be specified. organs to take into account. The possible dose that a certain volume

Evaluation and Approval of the Treatment Plan

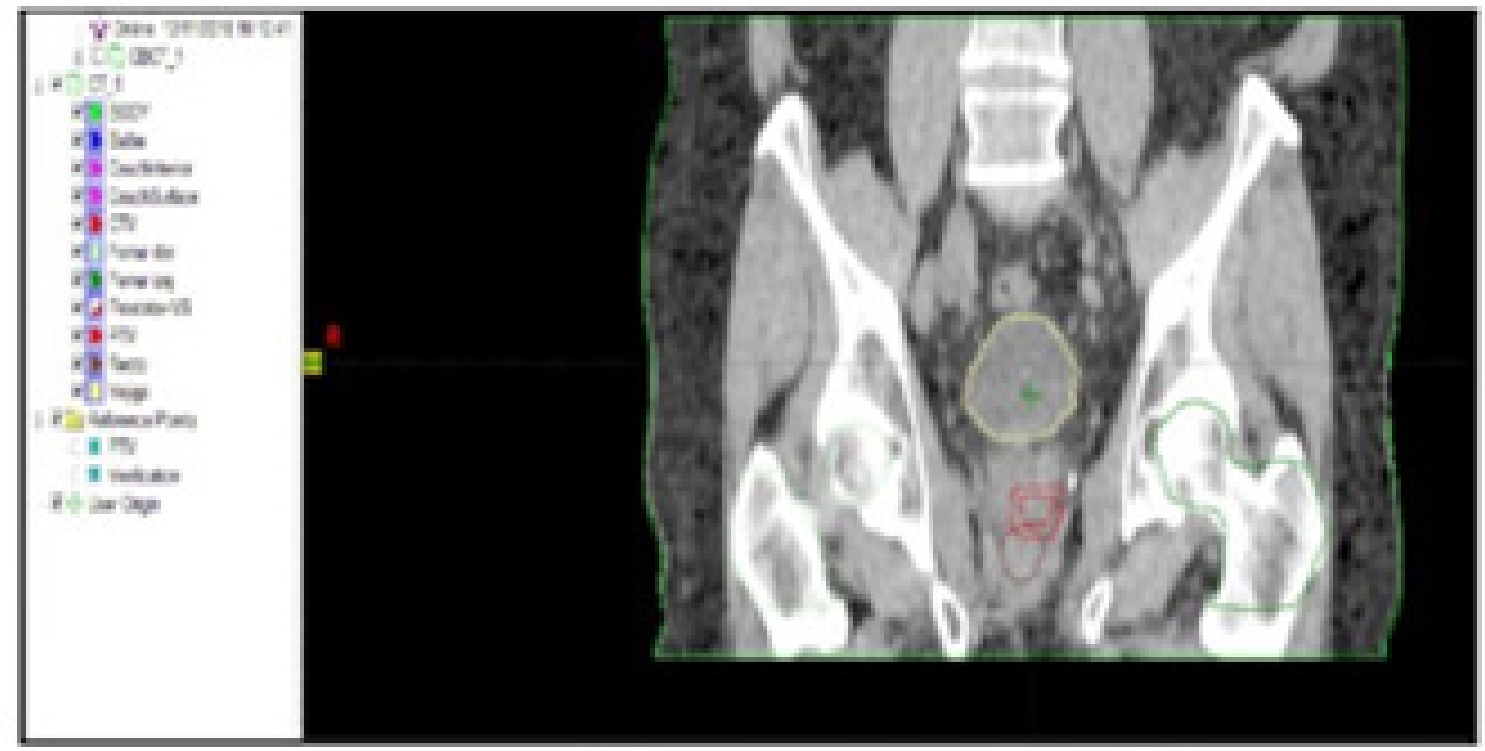

Figure 3: An Eclipse TM planning system, using modulated volumetric arcotherapy (VMAT) technique. 


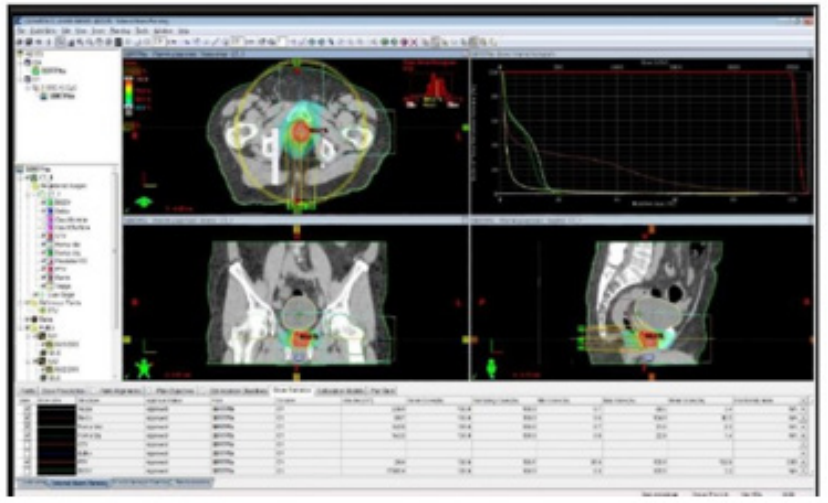

Figure 4: An Eclipse ${ }^{\mathrm{TM}}$ planning system, using modulated volumetric arcotherapy (VMAT) technique.

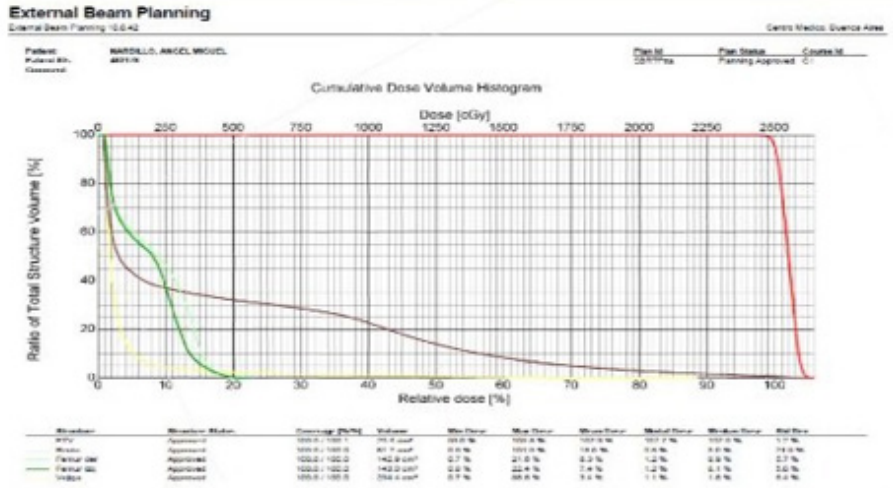

Figure 5: DVH.

Treatment planning will be carried out with an Eclipse тм planning system, using modulated volumetric arcotherapy (VMAT) technique. Once the medical physicist concludes the dosimetry, the plan proposed by the doctor will be evaluated, where the volume to be irradiated and the healthy organs will be observed, with the isodosis curves and the dose volume histograms (DVH) (Figures 3-5).

\section{Treatment Administration}

Figure 6: System Trilogy®. 


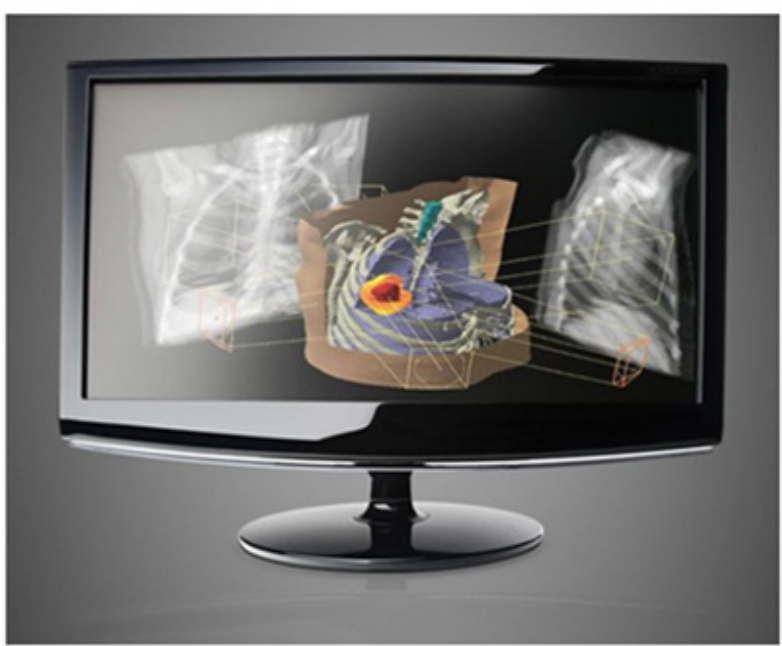

Figure 7: Eclipse treatment planning system.

The rescue treatment of prostate cancer recurrence will be performed in an advanced linear accelerator Trilogy® System, with SBRT technique (re-EBRT), with daily IGRT. Radiotherapy will be administered with modulated volumetric arcotherapy (VMAT). All products are from Varian Medical Systems, Palo Alto, California, distributed in Argentina by Photonix SRL, Autonomous City of Buenos Aires, systems, the technical characteristics of which have been published elsewhere [9,10] (Figures 6,7).

1. The patient must have the same preparation as that indicated on the simulation day,

2. The same immobilization is placed as that of the simulation tomography

3. An image of Cone-Beam Tc is made, which is analyzed by the oncological radiotherapist specialized in SBRT practices.

4. In case the doctor makes sure that the preparation or the positioning of the patient is not the correct one to carry out the application in a safe and precise way, we proceed to inform the patient, requesting what is necessary, in order to equalize the same condition as the day of the simulation, example in case it is required, it is requested to take more water, evacuate the rectum, etc.

5. The image is repeated, and the new condition is assessed with the image fusion performed with the Cone-Beam CT system.

A daily repositioning of the patient with correction for the movements of the inter and intrafraction organs are mandatory to improve the precision of the treatment.

\section{Toxicity and Effectiveness}

They will be evaluated according to the criteria of CTCAE (Common Terminology Criteria for Adverse Events) version 4.0.

\section{Possible toxicities include}

\section{Treble}

Urinary genital (GU): increased urinary frequency is the most common with urinary urgency. Will be managed with: Tamsulosin, consider routine prescription for 6 weeks after SBRT, longer as needed for symptom control.

Intestinal Gastro (GI): proctitis and diarrhea. It will be managed with: low-residue diet, antidiarrheals and rectal amifostine (Simone et al 2008).

\section{Chronicles}

1. Cystitis

2. Urethral stricture

3. Rectal ulcer

4. Erectile dysfunction

An increased risk of urethral stricture is observed if there is previous TURP, which is why it contraindicates SBRT.

\section{Tracing}

The follow-up of the patients will be performed with serum PSA level controls every 3 months during the first year, then PSA controls every 6-12 months, up to 5 years, and then annually until any biochemical or clinical progression. Control is suggested with the same study that detected the focus of local relapse, in case of progression. In patients with a reduction or stabilization of PSA levels at follow-up, it is not necessary to request additional evaluation of radiological or nuclear medicine.

\section{Conclusion}

The protocol developed has allowed us to correctly implement SBRT radiotherapy at the Hospital de Argentina, for the treatment 
of patients with previously irradiated prostate cancer who have relapsed locally. We believe that the treatment can be carried out safely and efficiently, with the fulfillment of each of the proposed points and with the appropriate equipment and with state-of-theart linear accelerators, which have an image-guided radiotherapy system, which ensures the parameters of precision needed.

Therefore, we understand that SBRT as a rescue for isolated local PCa recurrence seems to be a feasible rescue treatment and promises enough to warrant more extensive research to define the optimal selection of patients and the parameters of dose and volume. more adequate to ensure greater control and less toxicity.

\section{Acknowledgment}

None.

\section{Conflict of Interest}

The authors declare no potential conflicts of interest.

\section{References}

1. Orit Kaidar-Person, Ronald Chen (2018) Hypofractionated and Stereotactic Radiation Therapy. A Practical Guide. E-boock. Recuperado de.

2. Charles RP, Michael KB, Alan WP (2001) Evaluation and Treatment of Men with Biochemical Prostate-Specific Antigen Recurrence Following Definitive Therapy for Clinically Localized Prostate Cancer. Rev Urol 3(2): $72-84$.

3. Leroy T, Lacornerie T, Emilie B, Philippe N, Eric L (2017) Salvage robotic SBRT for local prostate cancer recurrence after radiotherapy: preliminary results of the Oscar Lambret Center. Radiat Oncol 12: 95.

4. Leszek M, Małgorzata SF, Marcin M, Bogusław M, Andrzej T (2018) Salvage CyberKnife-Based Reirradiation of Patients With Recurrent Prostate Cancer: The Single-Center Experience. Technol Cancer Res Treat.
5. Filippo A, Alba F, Berardino DB (2015) SBRT and extreme hypofractionation: A new era in prostate cancer treatments? Rep Pract Oncol Radiother 20(6): 411-416.

6. Simon SL, Bin ST Jiade J Lu, Tracey E Schefter Editors (2012) In: Stereotactic Body Radiation Therapy.

7. (2015) Retreatment for prostate cancer with stereotactic body radiation therapy (SBRT): Feasible or foolhardy?

8. Roach M 3rd, Hanks G, Thames H Jr, Paul S, William US, et al. (2006) Defining biochemical failure following radiotherapy with or without hormonal therapy in men with clinically localized prostate cancer: recommendations of the RTOG-ASTRO Phoenix Consensus Conference. Int ] Radiat Oncol Biol Phys 65(4):965-974.

9. https://www.varian.com/es-xl/oncology/treatment-techniques/external-beam-radiatio $\mathrm{n} / \mathrm{vmat}$

10. http://photonix.com.ar/portfolio-posts/trilogy-system/

11. Rajni AS, Igor JB, David AL, Mack R (2016) Handbook of Evidence- Based Stereotactic Radiosurgery and Stereotactic Body.

12. http://www.rtog.org/CoreLab/ContouringAtlases/MaleRTOGNormalPelvisAtlas.asp

13. Fowler JF (2010) 21 years of Biologically Effective Dose.Br. J Radiol 83 (991): 554-568.

14. Barbara A, Jereczek F, Damaris PR, Dario Z (2019) Salvage Re-Irradiation for Local Recurrent Prostate Cancer with Sbrt. publicado Fr J Radiol 92 (1094): 20180494.Reiche EM, Nunes SO, Morimoto HK (2004) Stress, depression, the immune system, and cancer. Lancet Oncol 5: 617-625.

15. Spiegel D, Giese-Davis J (2003) Depression and cancer: mechanisms and disease progression. Biol Psychiatry 54: 269-282.

16. Dhillon VS, Dhillon IK (1998) Chromosome aberrations and sister chromatid exchange studies in patients with prostrate cancer possible evidence of chromosome instability. Cancer Genet 100: 143-147.

17. Li GY, Yao KT, Glaser R (1989) Sister chromatid exchange and nasopharyngeal carcinoma. Int J Cancer 43: 613-618. 